\title{
Brownian dynamics of a microswimmer
}

\author{
Vladimir Lobaskin, ${ }^{1}$ Dmitry Lobaskin, ${ }^{2}$ and Igor Kulic ${ }^{3}$ \\ ${ }^{1}$ Physics Department, Technical University of Munich, D-85748 Garching, Germany \\ ${ }^{2}$ Bear Stearns International, 1 Canada Square, London, E14 5AD, UK \\ ${ }^{3}$ School of Engineering and Applied Sciences, Harvard University, Massachusetts 02138, USA
}

(Dated: August 14, 2021)

\begin{abstract}
We report on dynamic properties of a simple model microswimmer composed of three spheres and propelling itself in a viscous fluid by spinning motion of the spheres under zero net torque constraint. At a fixed temperature and increasing the spinning frequency, the swimmer demonstrates a transition from dissipation-dominated to a pumping-dominated motion regime characterized by negative effective friction coefficient. In the limit of high frequencies, the diffusion of the swimmer can be described by a model of an active particle with constant velocity.
\end{abstract}

Attention of physics community to the problem of swimming at low Reynolds numbers, which is relevant for the world of microorganisms, was attracted by Edward Purcell in seventies [1]. He formulated the basic principles of self-propulsion and suggested a variety of simple model microswimmers that would propel themselves in the Stokesian regime using non-reciprocal cyclic moves. The most famous of them, three-link swimmer, was solved analytically only recently [2]. As the Stokesian regime is characterized by the absence of time in the flow equations, the description of self-propulsion reduces to a purely geometrical problem of transformation of the microswimmer's body shape. The problem was solved for various nearly spherical objects, whose surface is deformed by a wave-like perturbation in the manner of ciliated microorganisms $[3,4,4,5,6,6]$. A number of other simple models performing one- or two-dimensional non-reciprocal moves as well as their swimming performance were discussed recently [8, 9 , 10].

Recent advances in micromanipulation techniques made it possible to construct artificial swimmers mimicking the bacterial and protozoal self-propulsion mechanisms. Most of these machines, however, are supposed to be driven by an external fields rather than ATP hydrolysis. The first working device imitating the flagellum beating and driven by an external magnetic field was reported recently [11]. Realistic implementations of DNA-based nanomachines using the ratchet principle were also suggested [12, 13]. Other directions in development of self-propelling micromachines is related to use of anisotropic environments, active surfaces or chemical reactions, whose mechanical response has an inherent asymmetry [14, 15, 16, 17, 18, 19]. The focus of these publications is the propulsion mechanism as such and the dynamics of a swimmer on long timescales is usually not addressed. One should note, however, that on the nanometer and micrometer scale the thermal fluctuations are expected to compete with the propulsion mechanisms and, therefore, the interplay between the swimming and dissipation processes is of great interest [21, 22, 23, 24, 25, 26]. In this work, we report on general dynamic properties of a microswimmer at finite temperatures.

For our study we chose a simple model swimmer, consisting of three spheres with their centers comprising an equilateral triangle. The distances between the spheres are fixed. To impose propulsion, we make the spheres spin via applied constant torque. We arrange the torque directions as shown in Fig. 1a so that the net torque is always zero. This constraint mimics the rotation due to internal degrees of freedom, as it would be in case of a microorganism swimming. At the same time, the algorithm allows us to keep control over the amount of supplied energy. The dynamics of the three-ball animals was modeled numerically using a hybrid molecular dynamics/ Lattice Boltzmann (LB) simulation method [27, 28]. The spheres were modeled with the raspberry setup (a tethered network of point particles wrapped around a big Lennard-Jones (LJ) bead) [28] with the radius $R=3$, while the links by the massless harmonic bonds of length $L=10$ of high spring constant between the ball centers. The length units are defined by the LB cell size. Most simulations were performed in a cubic box of side 40 units with periodic boundary conditions. The dynamic viscosity of the fluid in the LJ units was set to $\eta=2.55$ if not specified otherwise, and the temperature to $T=5$. To simulate the action of finite temperature, a fluctuating stress tensor technique was used and in addition stochastic random force was applied to each of the beads composing the swimmer [27]. The velocity of each bead $\mathbf{v}$ is then calculated from the following Langevin equation

$$
m \frac{d \mathbf{v}}{d t}=-\zeta(\mathbf{v}-\mathbf{u}(\mathbf{r}))+\mathbf{f}(t)+\mathbf{F}_{\text {ext }}+\mathbf{F}_{\text {int }}
$$

where $m$ is the bead mass, $\zeta$ the friction coefficient, $\mathbf{u}(\mathbf{r}))$ the interpolated fluid velocity at the particle position $\mathbf{r}, \mathbf{f}(t)$ is a Gaussian white noise force with zero mean, whose strength is given via the standard fluctuation-dissipation theorem in the absence of the external force, $\mathbf{F}_{\text {int }}$ the interaction force between different beads comprising the raspberry (see Ref. 28] for details), and $\mathbf{F}_{\text {ext }}$ the "external" force driving the sphere rotation. The magnitude of the external force was chosen to produce the desired spinning velocity $\boldsymbol{\omega}=\mathbf{T} /\left(8 \eta \pi R^{3}\right)$, where the net torque $\mathbf{T}=\sum_{i} \boldsymbol{\rho}_{i} \cdot \mathbf{F}_{i}$ is a sum of 

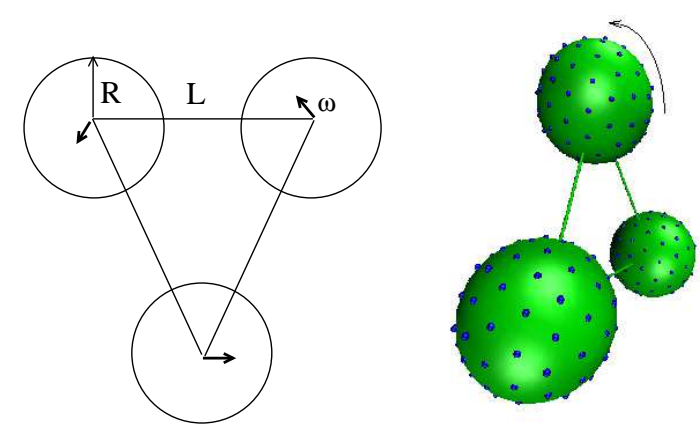

FIG. 1: The construction scheme of the three-ball swimmer: The balls are spinning around the in-plane axes so that the angular velocities $\boldsymbol{\omega}_{1}+\boldsymbol{\omega}_{2}+\boldsymbol{\omega}_{3}=0$. A simulation snapshot on the right hand side illustrates the raspberry model implementation of the swimmer.

the moments acting on each surface bead of the sphere, $\rho$ is the distance from the rotation axis to the $i$ th bead. The energy influx is then given by a sum $\varepsilon=\sum_{i} \mathbf{F} \cdot \mathbf{v}_{i}$ over all beads constituting the spheres or simply $\varepsilon=\mathbf{T} \cdot \boldsymbol{\omega}$.

Motion of the swimmer at zero temperature can be easily understood in the limit of small spheres and long links. Each sphere can be then represented by a rotlet 29]

$$
\mathbf{v}(\mathbf{r})=\frac{R^{3}}{r^{3}} \omega \times \mathbf{r}
$$

We have to find the flow field produced by two small spheres at the location of the third one and take just sum of their the components normal to the plane containing the three sphere centers, as the other components cancel. To have a zero net force acting on each of the spheres, it requires the sphere to move normally to the triangle plane with the velocity

$$
v_{0}=\sqrt{3} \omega R\left(\frac{R}{L}\right)^{2}
$$

We tested this prediction against simulation results for a swimmer with $R=3$ and $L=10$ (see Fig. 2). Despite the large ball size as compared to the link length, the equation gives a very good velocity estimate with relative deviation from the simulation data less than $4 \%$ up to $\omega=2$. The result given by Eq. (3) can be also be compared to the propulsion speed of a twirling ring, which corresponds to a generalization of the three-ball animal to the case of infinite number of balls filling a full circle of radius $r$ [12, 13]:

$$
v_{0}=\frac{R^{2}}{2 r} \omega\left(\ln \left(\frac{8}{R}\right)-\frac{1}{2}\right)
$$

The propulsion efficiency of this swimmer can be estimated as a relation of the energy dissipated in the forward thrust to the energy dissipated in the sphere spinning, which gives

$$
\beta=\frac{\mathbf{F}_{\mathrm{ft}} \mathbf{v}_{0}}{\mathbf{T} \cdot \omega}
$$

The steady state thrust force $\mathbf{F}_{\mathrm{ft}}$ is equal to the total friction force $\mathbf{F}_{\mathrm{f}}$ due to forward motion of the three-ball swimmer, which is related to the corresponding mobility as $F_{\mathrm{f}}=-v_{0} / \mu_{3}$. If the hydrodynamic interactions between the spheres are taken into account in the stokeslet approximation (the mobility of a pair of stokeslets to the first order in $r^{-1}$ is $\left.\mu_{2}=\frac{\mu_{1}}{2}\left(1+\frac{3}{4} \frac{R}{r}\right)\right)$, we get for the triplet

$$
\mu_{3}=\frac{\mu_{1}}{3}\left(1+2 \frac{3 R}{4 L}\right) \approx 0.63 \mu_{1}
$$

where $\mu_{1}=(6 \pi \eta R)^{-1}$ for the motion transversal to the triangle plane. The torque of the friction force exerted by a spinning sphere of radius $R$ is given by $T=8 \pi \eta R^{3}$. If we neglect hydrodynamic interactions (due to fast decay 


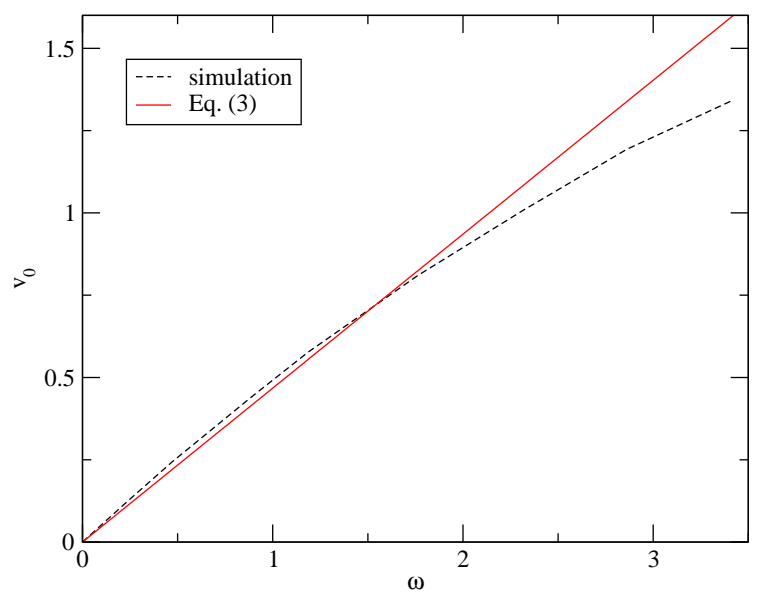

FIG. 2: Self-propulsion velocity of the three-ball swimmer with $R=3$ and $L=10$ as a function of the ball spinning frequency. The solid line was obtained using Eq. 3

of the the flow field magnitude for a rotlet, $(R / r)^{-3}$, it remains a good approximation for $\left.(R / L)<1\right)$, the energy dissipated just due to independent spinning of three spheres becomes $8 \pi \eta R^{3} \omega$. The efficiency is then $\beta \approx 0.5(R / L)^{4}$, which reaches its maximum of about $6 \%$ for contacting spheres.

We now study the motion of the swimmer at finite temperatures. Typical two-dimensional projections of the swimmer's trajectories observed at different ball spinning frequencies are plotted in Fig. 3. One can see that the character of the motion is changing significantly on increasing the frequency: the trajectories become more persistent with more extended straight segments and less abrupt changes of the direction. As a result, the swimmer with a higher $\omega$ travels over longer distances within the same time as compared with a less active one.

The mean-square displacements (MSD) of the swimmer's center of mass are shown in Fig. 彺 for four different spinning frequencies and three different temperatures. We see immediately, that the higher spinning frequencies correspond to a higher MSD, i.e. to the higher diffusivity. The shape of the MSD curves clearly indicates the existence of two distinct dynamic regimes: the driven motion at $t \leq 500$ where $\left\langle\Delta r^{2}\right\rangle \propto t^{2}$ and the diffusive motion at $t>1000$ where $\left\langle\Delta r^{2}\right\rangle \propto t$. In the case of high propulsion speeds, the transition from the deterministic driven motion to a random walk happens due to rotational diffusion of the swimmer. The "ballistic part" of the MSD coincides for the swimmers with the same frequency but different temperatures. In contrast, the random walk part is thermosensitive: higher temperature leads to a lower diffusivity. The transition between the two regimes happens on the characteristic timescale of rotational diffusion. The diffusion coefficient measured from the asymptotic behaviour of the swimmer at long times, $D_{\text {eff }}$, is plotted in Fig. $4 \mathrm{~b}$. We see that the measured diffusion coefficient grows nonlinearly with the drift velocity (or the spinning frequency as $v_{0} \propto \omega$ ).

A simplest model describing the diffusion of an active swimmer moving with a constant velocity $v_{0}$, whose direction of motion changes due to rotational diffusion with a diffusion constant $D_{r}$, gives the diffusion coefficient $D_{\text {eff }}=2 v_{0}^{2} / D_{r}$. This expression follows immediately from a reformulation of the known result for the end-to-end distance of a persistent polymer chain, which is $\left\langle\Delta r^{2}\right\rangle=2 l_{p} L, l_{p}$ being the persistence length and $L$ the contour length of the chain. In the swimmer variables, the persistence length of the trajectory is $l_{p}=\tau v_{0}$, where $\tau$ is the rotational correlation time, and its contour length is simply proportional to time: $L=t v_{0}$. Then, $D_{\text {eff }}=\left\langle\Delta r^{2}\right\rangle / 6 t=v_{0}^{2} \tau / 3$ [20]. The time $\tau$ is defined via the orientational correlation function of the swimmer $\langle\mathbf{n}(0) \cdot \mathbf{n}(t)\rangle$, where $\mathbf{n}$ is the normal vector to the plane of the triangle connecting the sphere centers. In case of random uncorrelated fluctuations, the correlation function decays at short times exponentially with time as $\langle\mathbf{n}(0) \cdot \mathbf{n}(t)\rangle=e^{-t / \tau}$. In case of an anisotropic rotation, one can expect that the decay is governed by the smallest correlation time, i.e. by the characteristic time of rotation in the direction with the smallest friction torque. The shortest orientational relaxation time expected for this swimmer corresponds to a rotation around the in-plane axis passing through the center of one ball and through the middle of the spring connecting the opposite ones. With a first order correction for hydrodynamic interaction between the balls we get

$$
\tau=\frac{1}{k_{B} T}\left(8 \pi \eta R^{3}+\frac{3 \pi \eta L^{2} R}{1-3 R / 2 L}\right)
$$




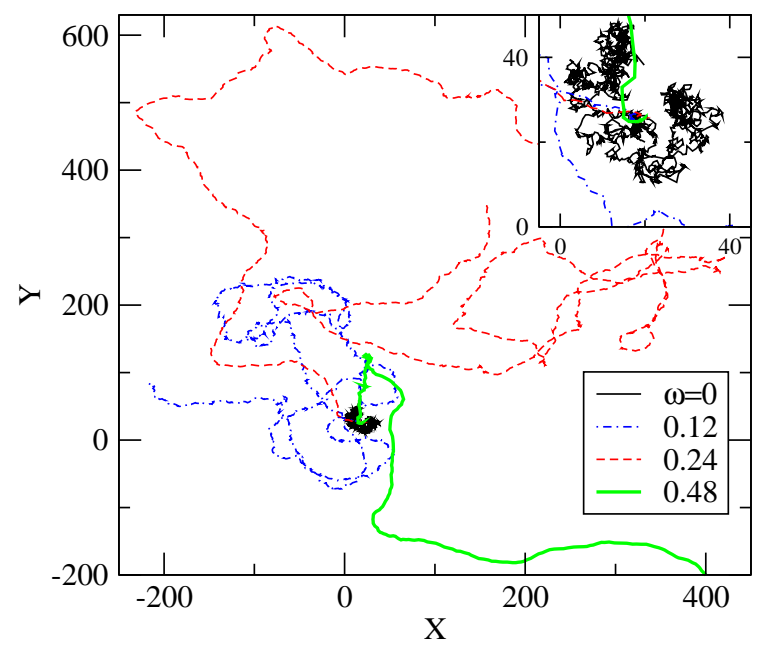

FIG. 3: Two-dimensional XY projection of the trajectories of the swimmer's center of mass position obtained with different ball spinning frequencies at $T=5$. The insert shows the magnified view of the initial part of the trajectories.

This formula gives $\tau=2250,1350$, and 675 for $T=3,5$, and 10 , respectively. This values are close to what we observe in simulation at low $\omega$. If we use this correlation time to predict the translational self-diffusion coefficient, the agreement with the measured data is convincing (the solid curves in Fig. 4b). We also tested our assumption regarding the orientational correlation time by direct measurement of the correlation function of the swimmer trajectories. From these measurements at low spinning frequencies we get $\tau=2300 \pm 200,1300 \pm 200$ and $700 \pm 100$ for $T=3,5$, and 10, respectively. These values are in good agreement with the estimated correlation times. In what concerns the assumption of constant velocity along the trajectory, we can check the swimmer's velocity distribution. The characteristic distributions of the velocity component perpendicular to the triangle plane (i.e. parallel to the thrust force) for $T=3$ are shown in Fig [5. While at $\omega=0.24$ the variance is comparable to the mean velocity, at the highest frequency, $\omega=1.2$, it does not exceed $25 \%$ of the mean. So, we see that the swimmer's velocity is narrowly distributed around $v_{0}$ and that also this assumption is justified. Thus, the swimmer's motion, especially at the high ball spinning frequencies (or energy influx rates) fits well the model of an active Brownian particle [21, 23], whose velocity is fairly constant in the magnitude while the direction is subject to thermal fluctuations. In other properties we observe more analogies to the active Brownian particle model. For example, the existence of a finite preferential velocity in our model is also reflected in the velocity distribution function shown in Fig. 5. Instead of a maximum at $v=0$ characteristic for systems in thermal equilibrium (the Maxwell distribution) it possesses two maxima at $\pm v_{p}$ and a minimum at $v=0$.

At $v_{0}^{2}>\left\langle v^{2}(\omega=0)\right\rangle$ we expect that the character of the swimmer's motion changes. As it was mentioned above, the characteristic velocity $v_{0}$ is a function of the energy influx rate, while the variance is fixed by the temperature and the swimmer mass: $\left\langle v^{2}\right\rangle=3 k_{B} T / M$, and characterizes the energy dissipation rate. Thus, the transition is expected at $\omega=k_{B} T L^{4} /\left(R^{6} M\right)$. The threshold values of the frequency are $\omega \approx 0.3,0.5$ and 1.0 (or $v_{0}=0.13,0.23$, and 0.45 ) for $T=3,5$ and 10, respectively. At the higher frequencies, other signatures of the driven regime can be observed, such as negative friction coefficient 23]. The friction coefficient can be extracted from the relation between the swimmer acceleration $\mathbf{a}$ and its instant velocity $\mathbf{v}$. We calculated these values by averaging over three velocity/acceleration components. The result for different spinning frequencies is shown in Fig. 66 top. As there is no other external forces acting of the swimmer, the acceleration is resulting from the viscous friction $a=F_{\mathrm{fr}} / M=-\gamma(v) v / M$. Here, the effective friction coefficient $\gamma(v)$ is a function of the instant velocity. The $a(v)$ curves for the lowest spinning frequency are fairly linear at low velocities, which corresponds to a constant positive friction coefficient. The curves bend toward smaller friction at the higher frequencies, and at the highest one all the curves cross zero and display a region of positive acceleration. All the $a(v)$ curves show two linear regions: one at $v \rightarrow 0$ and another one at the higher velocities. The corresponding nonlinear friction coefficient is plotted in Fig. 6 bottom. There we indeed notice saturation both at low velocities and at high $v$. While the limiting values $\gamma(v \rightarrow 0)$ shows strong dependence on the energy influx rate, the high velocity values seem to tend to a temperature independent limit, which only slightly decreases with the ball spinning frequency. We also notice that the parts of the curves with the acceleration parallel 

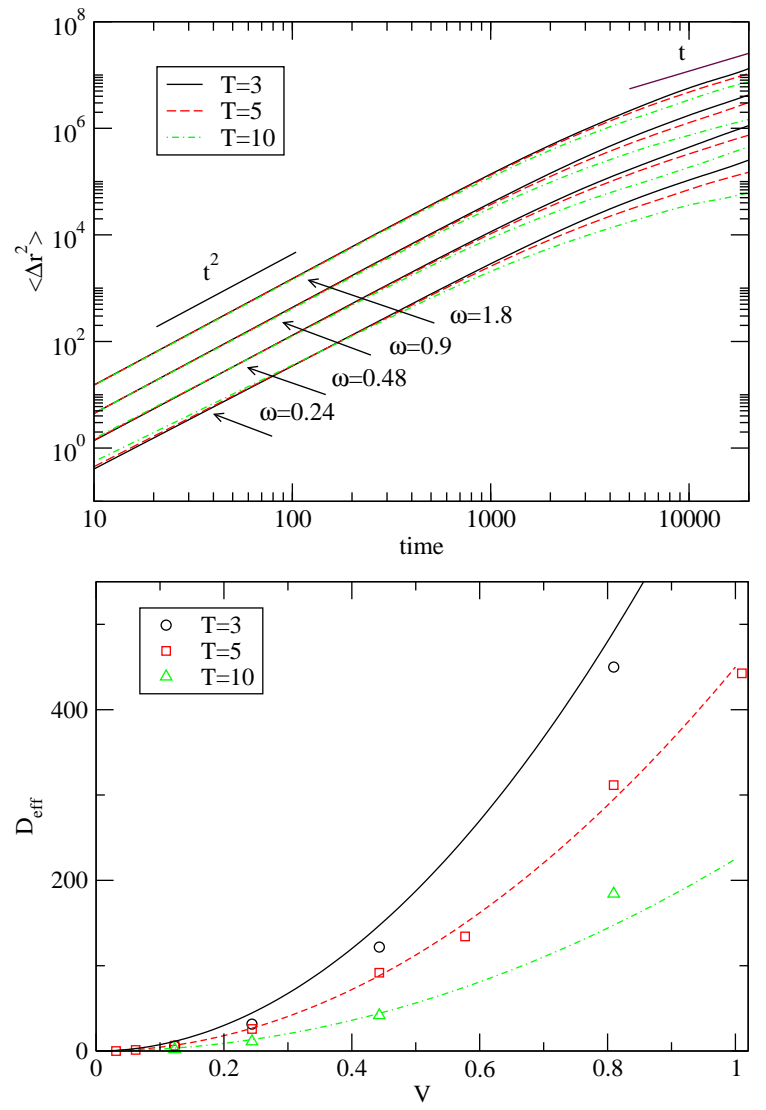

FIG. 4: Top: Mean-square displacement of the swimmer as a function of the ball spinning frequency and temperature. Bottom: Long-time diffusion coefficient of the swimmer at different temperatures measured from the long-time asymptotic behavior of the mean-square displacement. The solid lines are calculated as $D_{\text {eff }}=2 v_{0} / D_{r}$ (see the text for further details).

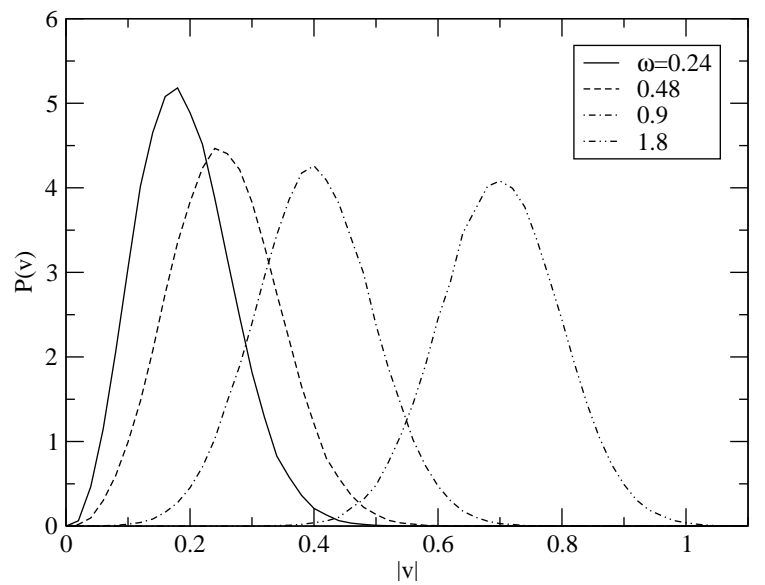

FIG. 5: Distribution function of the swimmer's velocity component in the direction of the thrust force at different spinning frequencies and temperature $T=3$. 

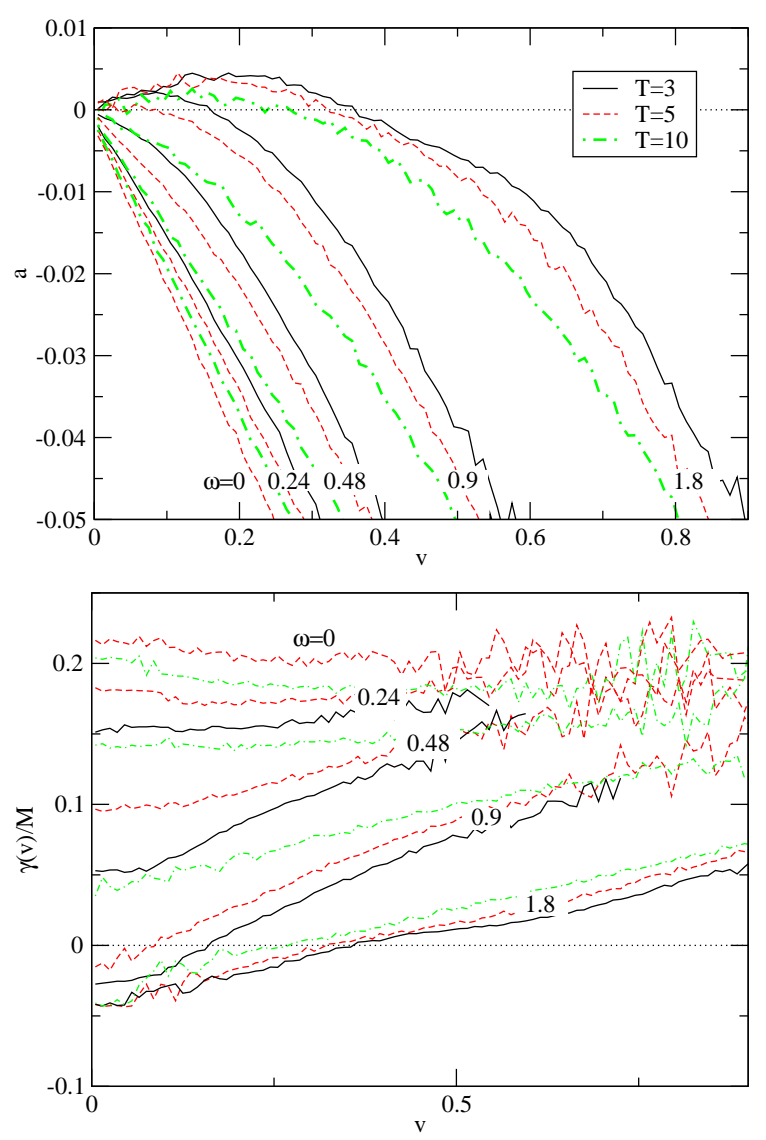

FIG. 6: Top: Swimmer acceleration as a function of its instant velocity at different ball spinning frequencies (marked on the curves) and temperatures. Bottom: The corresponding effective friction coefficient as a function of the swimmer's velocity.

to the instant velocity correspond to $\gamma(v)<0$. One can see that the transition into the driven regime with negative $\gamma$ occurs on increasing $\omega$. At $T=3$ and $T=5$ the negative friction appears at $\omega=0.9$ and 1.2 , at $T=10$ only at $\omega=1.2$, in agreement with the above estimates. The values $v_{p}$, at which the friction coefficient turns zero reflect therefore the preferential stationary velocity, at which the viscous resistance of the medium is exactly balanced by the swimmer's forward thrust.

We would like to note also that the behaviour of the friction coefficient qualitatively resembles the velocity dependence predicted within the active Brownian particle model [23]. In the adiabatic approximation the effective friction for a particle with an internal energy depot should vary as $\gamma(v)=\gamma_{0}-\frac{a}{b+v^{2}}$, where the coefficient $a$ is proportional to the energy influx rate. If applied to our case, the $\gamma_{0}$ would be the Stokesian viscous friction experienced by the swimmer. The negative values of the nonlinear friction coefficient can be observed provided that $\gamma(v)$ turns negative at finite velocities, which requires that $a>\gamma_{0}\left(b+v^{2}\right)$. We can see from Fig 6 at $0.24 \leq \omega \leq 0.9$ that the shape of the friction curves $\gamma(v)$ shows a similar behaviour.

Finally, we would like to note that the parameters of this system were chosen to demonstrate the wide range of dynamic properties. In case of a micrometer size living swimmers, the role of thermal fluctuations might be significantly smaller. Living microswimmers moving at the velocities of $10 \mu \mathrm{m} / \mathrm{s}$ would rather demonstrate the driven motion regime. Still, in presence of a stochasticity caused by internal mechanisms of the animal or spatial/temporal inhomogeneities of the medium, its competition with the deterministic drive would lead to qualitatively similar results. On the other hand, slow and small swimmers like the artificial ones, suggested in literature, would demonstrate many features described in this work even with thermal fluctuations. Another parameter that differentiates the dynamics studied in the present work from the biological microorganisms is the relatively high Reynolds number that reaches $\mathrm{Re}_{\text {max }} \approx 3$ for the highest velocities considered. So, the role of inertial effects is considerably overestimated as compared to the microworld. Our tests, however, showed that none of the reported properties can be attributed solely 
to inertia and we expect our predictions to be valid also for the typical microscopic objects.

We studied dynamics properties of a self-propelling microswimmer engine subjected to thermal fluctuations. Our model for the first time treats explicitly both the propulsion mechanics and fluctuations and demonstrates a rich interplay of these factors. Qualitatively, many of the observed properties (such as the non-linear velocity-dependent friction coefficient, characteristic crater-like velocity distributions at high energy influx rates, etc.) resemble those of the model of active Brownian particle [21, 23]. The differences related to the fluctuations of the propulsive effort, the broad velocity distributions, three-dimensional motion, and anisotropic friction, result in general in a more complex dynamic behaviour, which we hope will stimulate further theoretical effort in this field.

We are grateful R. Thaokar, W. Ebeling, L. Schimansky-Geier, U. Erdmann, B. U. Felderhof, J.-F. Joanny for stimulating discussions.

[1] E. Purcell, Am. J. Phys. 45, 311 (1977).

[2] L. E. Becker, S. A. Koehler, H. A. Stone J. Fluid Mech. 490, 15 (2003).

[3] H. A. Stone, A. D. T. Samuel, Phys. Rev. Lett. 77, 4102 (1996).

[4] A. Shapere and F. Wilczek, Phys. Rev. Lett. 58, 2051 (1987)

[5] A. Shapere and F. Wilczek, J. Fluid Mech. 198, 557 (1989).

[6] B. U. Felderhof and R. B. Jones, Physica A 202, 94 (1994).

[7] B. U. Felderhof and R. B. Jones, Physica A 202, 119 (1994).

[8] A. Najafi, R. Golestanian, Phys. Rev. E 69, 062901 (2004).

[9] J. E. Avron, O. Gat, O. Kenneth, Phys. Rev. Lett. 93, 186001 (2004).

[10] U. B. Felderhof, Phys. Fluids 18, 063101 (2006)

[11] R. Dreyfus, J. Baudry, M. L. Roper, M. Fermigier, H. A. Stone, J. Bibette, Nature 437, 862 (2005)

[12] I. M. Kulić, R. Thaokar, H. Schiessel, Europhys. Lett. 72, 527 (2005).

[13] I. M. Kulić, R. Thaokar, H. Schiessel, J. Phys.: Condens. Matter 17, S3965 (2005).

[14] S. Camalet, F. Julicher, J. Prost, Phys. Rev. Lett. 82, 1590 (1999).

[15] Y.W. Kim and R.R. Netz, Phys. Rev. Lett. 96, 158101 (2006).

[16] P. E. Lammert, J. Prost, R. Bruinsma, J. Theor. Biology 178, 387 (1996).

[17] R. Golestanian, T.B . Liverpool and A. Ajdari, Phys. Rev. Lett. 94, 220801 (2005).

[18] N. Mano and A. Heller, J. Am. Chem. Soc. 127, 11574 (2005).

[19] W. F. Paxton, A. Sen, and T. E. Mallouk, Chemistry - A Eur. J. 11, 6462 (2005).

[20] P. S. Lovely, F. W. Dahlquist, J. Theor. Biol. 50, 477 (1975).

[21] F. Schweitzer, W. Ebeling, and B. Tilch, Phys. Rev. Lett. 80, 5044 (1998); Phys. Rev. E 64, 02110 (2001)

[22] W. Ebeling, F. Schweitzer, B. and Tilch, BioSystems 49, 17 (1999).

[23] U. Erdmann, W. Ebeling, F. Schweitzer, and L. Schimansky-Geier, Europhys. J. B 15, 105 (2000).

[24] T. Vicsek, A. Czirók, E. Ben-Jacob, and I. Cohen, Phys. Rev. Lett. 75, 1226 (1995)

[25] I. Derényi and T. Vicsek, Phys. Rev. Lett. 75, 374 (1995)

[26] T. Vicsek, Fluctuations and scaling in biology, (University Press, Oxford, 2001).

[27] P. Ahlrichs, B. Dünweg, Int. J. Mod. Phys. C 9, 1429 (1998); J. Chem. Phys. 111, 8225 (1999)

[28] V. Lobaskin, B. Dünweg, New J. Phys. 6, 54 (2004).

[29] L. D. Landau and E. M. Lifshitz, Hydrodynamics (Pergamon, New York, 1959). 